MATEC Web of Conferences 22,04008 (2015)

DOI: $10.1051 /$ matecconf/ 20152204008

(C) Owned by the authors, published by EDP Sciences, 2015

\title{
Application of Remote Sensing Technology in Mine Environment Mon- itoring
}

Yue Li

Chinese Academy of Geological Sciences, Beijing, China

Hongli Zhao \& Jinghui Fan

Beijing Land Resources Information Development Research Laboratory, Beijing, China

\begin{abstract}
Mine environment problem caused by the exploitation of mineral resources has become a key factor which affects normal production of mine and safety of ecological environment for human settlement. For better protection and management of mine environment, this article has introduced the important role of remote sensing technology in pollution monitoring of mine environment, geological disaster monitoring and monitoring of mining activities.
\end{abstract}

Keywords: mine environment; remote sensing technology; environmental monitoring

\section{INTRODUCTION}

Engineering, technical and economic activities of human will lead to specific environmental and geological problems under certain geological environment [1]. Geological environment of mine is an organic whole of environment and resources, in which mineral resource is the material basis for human survival and development while environment is the necessary condition for human survival and development. Environment and resources will interact and interconvert with each other under certain condition. Irrational exploitation of mineral resources will inevitably destroy geological environment of mine, and deterioration of geological environment will in turn destroy mine resources (land resources, landscape resources and water resources and so on of mine lot). The only scientific development is rational exploitation of resources coordinated with environmental protection [2]. In the meantime when human exploit and utilize mineral resources to promote their own survival and development as well as social progress and material prosperity, notable influence is made to geological environment of mines even with severe cases such as resources damage, geological disaster and pollution of mine environment [3].

Environmental pollution caused by mine exploitation is a complicated and systematic change, the tendency of which cannot be indicated by any single factor and instantaneous indication indicator [4]. Emphasis on strengthening engineering, environmental and geological survey of mine exploitation has evoked concern of the whole society. Effective monitoring of mineral resources exploitation and caused ecological environment problem thereof is the primary premise to enhance probability of sustainable development of mine enterprises. Monitoring of geological environ- ment of mine is crucial to improving mine environment and reducing and mitigating damage caused by exploitation. Leaders of departments concerned as well as scientific and technological workers have been looking for a rapid and reliable monitoring method, to grasp the situation of national mine exploitation in real-time and efficiently. With continuous development of remote sensing technology, this desire is becoming reality gradually [5].

With its macro-characteristics, authenticity and comprehensiveness, remote sensing image has provided us with a reliable basis for identification and analysis of landforms, geological structures and land features, which has incomparable advantages than other methods [6]. With continuous development of satellite remote sensing technology, spatial resolution and spectral resolution of commercialized resource satellite data are becoming increasingly higher, and it has become a necessary tendency to use this technological means to monitor industrial and mining district [7].

\section{OVERVIEW OF REMOTE SENSING TECH- NOLOGY}

\subsection{Concept of Remote Sensing Technology}

Research group of the United Nations using satellite to perform remote sensing on the earth defines remote sensing as "Electromagnetic emission at certain wavelengths of observed object or near-earth object from ultraviolet ray to microwave". To be specific, remote sensing is to use modern carrier, electronic and optical instrument in an active or passive manner to receive and process electromagnetic wave of certain passable wavelengths from ultraviolet ray to micro- 


\section{MATEC Web of Conferences}

wave transmitted or reflected by research object at earth surface or somewhere deeper, thus to obtain relevant information of research object, realizing reception, transmission, processing and application processing of entire information of the detection object Emerging with advent of space technology, remote sensing technology belongs to space science and is called "eye" of the universe. Space remote sensing technology is the main body of remote sensing technology [8]

Remote sensing technology is widely used in detection of earth resources, prediction of earthquake and volcanic eruption, monitoring of environmental pollution as well as departments such as metallurgy, geology, oil, agriculture, forestry, water conservancy, surveying and mapping, weather, ocean and so on. Remote sensing technology is superior with characteristics of wide range, fast speed and low cost in monitoring and is convenient for long-term dynamic monitoring, etc. In mine monitoring, remote sensing data such as MSS, LandsatTM, ETM, SPOT, IKINOS, QUICKBIRD and so on are commonly used [9].

\subsection{Remote Sensing Monitoring}

Development of satellite remote sensing has made it possible for monitoring of mine ecological environment based on high resolution satellite remote sensing image to meet actual demand with adequate accuracy. Various ecological environment factors can be fully reflected on the remote sensing image by meter-scale spatial resolution, digital image processing is performed based on spectral feature and imaging feature of various ecological environment factors, pollution investigation and analysis of environmental elements are conducted on the basis of information extraction and classification to acquire pollution and damage situation of each environmental element as well as overall situation of regional ecological environment, and to analyze major pollution and its distribution, dispersal path of pollution and so on in combination with information affected by remote sensing. Comprehensive, real-time and abundant information source of mine ecological environment can be acquired through processing of remote sensing image, to support decision-making on environmental control and to evaluate control effect. With regard to ecological restoration and reconstruction of mine, satellite remote sensing will also play an increasingly important role. [10]

As one of the other important tools to obtain remote sensing data, UAV remote sensing is high in imaging resolution and flexible in data acquisition and so on. With aerial photo acquired by UAV, methods such as indoor interpretation combined with field investigation, remote sensing data combined with existing information, field spot check and expert assessment and so on are used to enhance quality and accuracy of result. UAV remote sensing technology has gained more and more popularity in investigation and monitoring of mine geological environment along with its continuous development. [11]
During data processing, geometric correction is performed first on acquired data to eliminate extrusion, distortion, extension and excursion of image data; image enhancement is used second to enhance interesting feature of remote sensing image data and to deblur image at the target section, thus to improve interpretation ability; after which remote sensing interpretation can be conducted for various ecological environmental elements in the research area to get required environment information.

\section{APPLICATION OF REMOTE SENSING IN MINE MONITORING}

\subsection{Pollution Monitoring}

\subsubsection{Air}

Taking coal mine as example, large amount of $\mathrm{CO}_{2}$ and $\mathrm{CH}_{4}$ and so on are emitted into the air directly during coal exploitation. Among which, $\mathrm{CH}_{4}$ will not only lead to more strong greenhouse effect than $\mathrm{CO}_{2}$, but also will cause destruction of the ozone layer in case of excess emission. Coal exploitation will also produce a large amount of coal gangue, which contains sulfide and may combust spontaneously in case of long-term stacking, emitting a large volume of hazardous gas such as $\mathrm{SO}_{2}, \mathrm{H}_{2} \mathrm{~S}, \mathrm{CO}_{2}$ and so on [12]. In addition, it is inevitable that a lot of dust is emitted into the air during mine production. Waste rock and tailings emitted by mine will also produce a lot of dust under wind force and pollute fresh air [13].

Ground remote sensing technology is used to monitor air pollution source, observing emission intensity of air pollutants, dust concentration and site of the mine simultaneously through multi-spectral means. Remote sensing technology uses multi-machine spectrum to perform synchro-photographing, records emitted dust on specially made multi-spectral film on the basis that different physical properties of different substances may have different spectral features, which causes substances with different properties within the same object to be selectively recorded on films with different band values, after which multi-spectral digital system is adopted with matrix additive color synthesis and multi-dimensional level segmentation subtraction to acquire digital image and multiple groups of data, and generation analysis, clustering analysis and piecewise regression are conducted on these data to realize semi-quantitative estimation of air pollution concentration [8].

\subsubsection{Water}

Wastewater produced by mining every year in our country accounts for about $5 \%$ of total amount of discharged industrial wastewater, and a large volume of untreated wastewater is discharged into rivers, lakes and seas, causing severe pollution. Among which, cooper mine causes the most severe pollution, with wastewater mainly of acid wastewater (from waste dump and open pit) and alkaline wastewater (from 
dressing plant) [14]. As for waste slag and tailings stacked in large quantity at the mine, large amount of slime water and acidic water containing heavy metal will bleed out under long-term weathering, leaching and leakage even after the pit is closed, causing severe pollution of ground waters and underground water sources [15]. In acid mine water, $\mathrm{Fe}^{3+}$ and $\mathrm{Al}^{3+}$ will generate amorphous oxide particles which are important carriers for adsorption migration of metal in polluted water due to hydrolytic precipitation. Acid wastewater of mine contains large amount of heavy metal as well as poisonous and hazardous elements (such as cooper, lead, zinc, arsenic, hexavalent chromium, mercury, cyanide and so on), which will dissolve out some heavy metal ion and poisonous pollutants under long-term soaking and scouring of rainfall and penetrate into ground water zone or generate surface runoff polluted water, causing large-area pollution of the mine [16].

Research of water environment on the image through remote sensing method is mainly based on color, shape and so on of water presented on the image. At present, it is still difficult to determine chemical index of water directly from image, however, hues and levels of water has reflected pollution status to some extent, which can be used to qualitatively determine water quality pollution in combination with field investigation and chemical analysis completed by predecessors. $\mathrm{Lu} \mathrm{Xia}$ and others [17] utilize multi-spectral remote sensing technology and ground hyper-spectral measurement technology to survey and analyze status and extent of water pollution at the mine, and have reached a conclusion: spectral reflection curves of different polluted water have large difference within visible range. However, in general, water under acidic pollution has strong red light reflection while water under alkaline pollution has strong green light reflection; spectral angle mapper technology can be used to obtain water pollution information of the whole mine based on spectral curve analysis of measured water.

\subsubsection{Vegetation}

Excavation of strip mine requires occupying large area of land, leading to decline of underground water level of the whole surrounding area, which will cause water shortage of surface soil, sandification and desertification of land and affect vegetation growth shape, such as chlorosis and dehydration of leaf and weakening of vegetation growth. Various barren rock, tailings and other wastes generated by mining contain large amount of hazardous substances which will penetrate into soil under raining, weathering and seepage after long-term stacking, leading to pollution of soil base and deterioration of soil structure [13], which will cause injury spot on vegetation leaf and quality degradation of products, and so on. Coal exploitation will also produce a large amount of coal gangue, which contains sulfide and may combust spontaneously in case of long-term stacking, emitting a large volume of hazardous gas such as $\mathrm{SO}_{2}, \mathrm{H}_{2} \mathrm{~S}, \mathrm{CO}_{2}$ and so on to the air [12], which will cause acid deposition pollution and a series of negative ecological effects on vegetation. For example, undesirable changes take place on material composition (such as sulfur content increase, chlorophyll content) and cellular structure of vegetation.

It is known to all that reflective capacity of vegetation leaf to visible light (0.38um 0.76um) mainly depends on chlorophyll. While at near-infrared band (0.76um 1.0um), reflective capacity of vegetation mainly depend on cellular texture and structure of vegetation. Therefore, physiological changes above of polluted vegetation will inevitably lead to changes of reflection spectral features of the leaf, which will be reflected on features of the remote sensing image. This is the theoretical basis for remote sensing monitoring of affected situation of polluted vegetation. Specifically, reflectivity of vegetation under normal condition for electromagnetic wave of near-infrared band is strong while that of the polluted vegetation is decreased rapidly. Therefore, magnitude of vegetation reflectivity of near-infrared band has reflected vegetation ecological environment and pollution situation of a region to some extent [8], thus to deduce severity of pollution due to mine exploitation in the region indirectly.

Mine pollution mainly include water pollution, soil pollution and air pollution, among which, water pollution can be identified directly by remote sensing technology while air pollution and soil pollution are hard to be identified directly by remote sensing technology, which can be used to identify vegetation pollution, thus to reflect air pollution and soil pollution indirectly. At present, there are mainly 3 methods for identification of mine vegetation pollution, which are image compounding method, vegetation index method and vegetation greenness method respectively. Pollution situation of mine vegetation can be roughly identified by processing and comparison of two data source on vegetation pollution obtained through vegetation pollution identification based on ASTER and vegetation pollution identification based on QuickBird. However, vegetation index method and vegetation greenness method are subject to influencing of shades of clouds and mountain and human production activities on identification of mine vegetation pollution, and thus attention shall be paid to eliminate interference in this aspect during actual processing. Meanwhile, vegetation greenness method shall be adopted with ASTER and other low-resolution data sources for large-scale identification of mine vegetation pollution considering project cost and so on. Whereas, vegetation index method shall be adopted with QuickBird and other high-resolution data sources for small-scale typical mine. [18]

\subsection{Monitoring of Geological Disaster}

Mine geological disaster means mine lot disaster generated by mining and production activities of human which destroys geological environment, endangers safety of life and property and brings about heavy 


\section{MATEC Web of Conferences}

economic losses ([19]; [20]) According to statistics, exclusive of earthquake and volcanic eruption, 15 main geological disasters related with human activities such as landslide, mud-rock flow, ground fissure, surface subsidence and so on have caused direct economic loss of about an average of RMB 30 billion yuan in China every year ([21]; [22]). Goaf developed by underground exploitation of mine is easy to cause surface collapse, fissure and subsidence.

Electromagnetic wave information received by satellite remote sensing reflects spectral feature and geometric feature of surface material [23]. Magnitude of spectral reflection energy received by sensor relate not only to surface spectral features, but also to dimension of incident angle of light. With difference of reflection energy of the same surface feature due to topographic changes of gradient and slope aspect, we can identify fluctuant change of topography on the remote sensing image. Geological disaster caused by exploitation of underground mineral resources has changed geometrical morphology and spectral features of surface. Generated faint information change has left traces on the remote sensing image, laying foundation for remote sensing identification research of geological disaster. For relatively large-scale geological disaster, remote sensing is undoubtedly the optimum observation method [24].

Collapse pits on remote sensing image present as distinct annular or oval spots and plaques, with clumped distribution of independent entity in different hues and shades. Vegetation inside the pit shows a reddish color. As a negative relief with certain depth, collapse pit has distinguished three-dimensional effect under shade. Compared with three-dimensional effect of positive relief (such as grave and independent crown), the three-dimensional effect of the negative relief is just the opposite. Shade of collapse pit appears at lower half inside the annular pattern spot while that of the mound appears at upper half inside the annular ring spot, which is an important sign of correctness for determination of collapse pit [24] Surface collapse presents as gray, dark gray and black in hue; exists at mountain top, hillside and foot in position; appears usually as round in shape, while those with more surface collapse appear as bead-like distribution; its diameter ranges from sub-meter to several meters; and the slope usually presents as light blue or dark blue due to artificial interferences [25].

Ground subsidence on the remote sensing image has a distinguished feature that irregular closed and semi-closed girdle or strip image, sometimes intermittent banding with certain altitude difference, width of $1 \sim 2 \mathrm{~m}$ and length of dozens of meters take shape at edge of the depression area. Hue at upward side (sunny slope) of the girdle is brighter while that of the downward side (shady slope) is darker, this kind of shade and color differences are caused by negative relief due to subsidence, which has resulted in sudden changes of topographic gradient and slope aspect, and has changed incident angle of light, leading to change of local spectral reflection energy. Affected by goaf area, subsidence area has certain width with area differing from several hundred square meters to several tens of thousands square meters. Change of microrelief can be used to infer location of ground fissure, which can be taken as basis to delineate and calculate area of subsidence area. [24]

Collapse on the remote sensing image presents as blending of white and light blue in hue, exists usually at mountain area with steep terrain, appears as funnel-form with sheet distribution and comparatively large overall area; and the artificial interference is relatively weak [25]. While ground fissure presents as dark gray and black in hue, exists usually at mountain top and hillside with high altitude; appears as slim strip distribution in shape with width ranging from several centimeters to over 10 meters and length ranging from several meters to several hundred meters; and the slope usually presents as light blue or dark blue due to artificial interferences [25].

\subsection{Monitoring of Mine Exploitation Activities}

With sustained and rapid development of economy in China, demand of all walks of life for resources is increasing day by day, which has caused increase of intensity for resource exploitation. However, issues such as prospecting and exploitation without license, cross-border exploitation and illegal transfer of exploration right and so on occur repeatedly during mineral resources exploitation, mining without regulations or plans, wasting of resources and destruction of environment and so on are relatively severe in certain regions, causing a series of geological disasters and hazards such as land occupation, pollution, landslide, subsidence and so on, or even dam collapse of tailings pond, which results in casualties and property losses Dynamic monitoring of mineral resources exploitation can be achieved by many methods; among which remote sensing is a comparatively mature monitoring means that is capable of reflecting various situations at the exploiting area in a more intuitive manner [26].

High-resolution satellite data (QuickBird, IKONOS) can display very small-scale mining activities; it is very effective to monitor mining without regulations or plans during primary stage. Professionals can determine stacking status of solid wastes, exploitation situation of mine (under exploitation or already closed) and so on according to the image. It is available to determine whether illegal exploitation exists (such as cross-border exploitation, one license for multiple mines and so on) at the mine according to approved mining boundary. This data has very wonderful effect on monitoring of geological disasters such as ground fissure, surface collapse and so on, and thus it makes an ideal data source. However, it is only suitable for small-range monitoring of key area due to relatively high price. Medium-resolution data can show open-pit mine, tailings pond and large-scale solid waste pile.

SPOT-5 satellite image can show general-scale mining activities. Such as exploitation point location of carven mine, various scales of solid waste piles as well as certain scale ground fissure, mine building and 
traffic and so on. However, it fails to provide a satisfactory show of exploitation situation of small-scale cavern mine [7].

In addition, vegetation index can be used to monitor mine expansion. Vegetation index is a simple and effective measure parameter used in remote sensing to represent surface vegetation coverage and growth status [27], and is widely used in qualitative and quantitative assessment of vegetation coverage and growth vitality as the most important data source to reflect surface vegetation information [28]. Vegetation index has certain indicative meaning on vegetation growth, biomass and so on, and is a significant index to infer biomass and productivity, and to evaluate structural and functional features of ecosystem, and so on. Gan Puping and others [29] divided vegetation pollution degree correspondingly according to maximum absorption depth of vegetation at location near $685 \mathrm{~nm}$. Specific to effect of mine exploitation on vegetation, Qi Xiaoying and Yan Mingxing [30] put forward to calculate soil-atmospherically resistant vegetation index (SARVI) through remote sensing image to reflect mine expansion indirectly, which shall then be analyzed in combination with mine expansion status acquired by supervised classification of remote sensing, thus obtaining mine expansion information within research area in a faster and better manner.

\section{CONCLUSION}

Under current situation, remote sensing technology has become an indispensable means used in mine environment monitoring. With gradual improvement of remote sensing technology and theory and continuous enhancement of spatial resolution, temporal resolution and spectral resolution of remote sensing image, master of application of remote sensing technology means master of its advantages such as macrocosm, comprehensiveness, speediness, dynamics, accuracy and timeliness, and will lead to important achievements in mine dynamics monitoring and many other aspects. Remote sensing technology will certainly become one of the indispensable means in geological works of mine. In line with respective industrial characteristics and environment management requirement, each mine enterprise can carry out environment monitoring according to local conditions, complete monitoring projects gradually, improve monitoring techniques continuously, strive to realize standardization, normalization and scientification of environment monitoring of the enterprise, to provide powerful technical assurance for mine enterprises to transform traditional development pattern and move towards new type of industrialization path.

\section{REFERENCES}

[1] Ha Chengyou, He Qingcheng. \& Jai Xuelang. 2002. Environmental geology: development and challenge. Geological Bulletin of China, (3).

[2] Jiang Chengsong. 2004. Elaborate environmental geology disciplines to improve environmental geology. Geological Bulletin of China. 23 (8).

[3] $\mathrm{Xu}$ Youning. 2008. Current situation and outlook for investigation of mine geological environment. Geological Bulletin of China. 27(28).

[4] Yan Chun, Liu Suhong. \& Zhao Xiang. 2005. A study on the synthetic evaluation index of the mine environment remote sensing. Remote Sensing Information. (6).

[5] Shang Hongying, Chen Jianping, Li Chenzun. \& Wang Yuntao. 2008. Application of RS in mine dynamics monitoring--A case study of rare metal mine concentration area. Remote Sensing Technology and Application, 23(2), (4).

[6] Chen Jifu. 2006. Application of RS technique in mining geology. Friend of Science Amateurs.

[7] Wang Xiaohong, Nie Hongfeng, Li Chengzun. \& Wang Jin. 2006. Application of different data sources in the investigation of exploitation situation and environment of mines. Remote Sensing for Land \& Resources. 68(2).

[8] Feng Kailong. \& Ma Guoxin. 2007. Application of remote sensing technology in environment monitoring and its principle. Collected Papers of the First Session Academic Conference of Communication Faculties of Chinese Universities.

[9] Cheng Ting, Chen Zhihua. \& Lu Xiaohui. 2006. Application of remote sensing technology in mine environment monitoring--A case study of eastern Hubei. $\mathrm{Na}$ tional Environmental Ecology and Geochemistry Investigation and Assessment Abstracts Set.

[10]Du Peijun. 2001. Development of high-resolution satellite remote sensingand its application in mines. Coal, $10(1)$.

[11]Zhou Wensheng, Wu Zhenyu. \& Liu Haiyan. 2014 Application of UAV remote sensing in investigation of mine geological environment. Ground Water.

[12]Li Xiangyi. \& Li Zhongxue. 2006. Economics of mining industries. Beijing: Metallurgical Industry Press.

[13] Sun Zhiwei. 2008. Countermeasure suggestions for resolving mine environmental problems in China. Resources and Industries, 10(1).

[14] Xu Wanwen. \& Zhang Wentao. 2004. Analysis of acid mine drainage pollution in Dexing Copper Mine. Jiangxi Chemical Industry, (1).

[15]Yin Xianglin. 2003. Discussion on geology calamity problems in the mine environment and countermeasures Conservation and Utilization of Mineral Resources, (8).

[16]Liu Zheng, Zhao Xuyang. \& Dang Hongyuan. 2012. Research progress on remote sensing monitoring of water and soil environment of mine exploitation. Journal of Shijiazhuang University.

[17]Lu Xia, Liu Shaofeng, Hu Zhenqi. \& Lin Yan. 2006 Study on remote sensing identification of water pollution in a mining area. Mining Research and Development. 26(4). 


\section{MATEC Web of Conferences}

[18]Huang Tielan, Wang Gengming, Li Wensheng. \& Zhu Junfeng. 2014. Study on remote sensing identification methods for vegetation pollution information of Dabaoshan mining area in Guangdong. Journal of Geology.

[19] Tang Zhongli, Li xiaohu, Jiao Jiangang and so on. Geological environment problems of mine and prevention countermeasures. Journal of Earth Science and the Environment.

[20]Li Shulin. 2002. On necessity to carry out engineering geological disaster research of metal mine. Mining Technolog.

[21] Tu Huaikui. 2000. Type and distribution characteristics of geological disasters in China. Mineral Resources and Geology. 14 (2).

[22]Hu Huasheng. \& Tang Xianwu. 2000. Geological environment expects our protection. China Land and Resources News, (1)

[23] Chen Shupeng, Tong Qingxi. \& Guo Huadong. 1998. Mechanism of remote sensing information. Beijing: Science Press.

[24]Li Chengzun, Nie Hongfeng, Wang Jin. \& Wang Xiaohong. 2005. A remote sensing study of characteristics of geological disasters in a mine (1).

[25] Wang Qinjun, Chen Yu. \& Lin Qizhong. 2010. High resolution remote sensing method study of geological disasters in mines--A case study of Shijiaying Mine in Fangshan, Beijing. Journal of Institute of Disaster Prevention.

[26]Cai Dongmei, Li Mingzhe. \& Cao Jiru. 2011. Application review of remote sensing technology in exploitation dynamics monitoring of mineral resources. Mine Surveying.

[27]Guo Ni. 2003. Vegetation index and its research development. Journal of Arid Meteorology, 21(4): 71-75.

[28] Tian Jing, Yan Yu. \& Chen Shengbo. 2004. The advances in the application of the remote sensing technique to the estimation of vegetation fractional cover. Remote Sensing for Land \& Resources. (1).

[29] Gan Puping, Liu Shengwei. \& Zhou Qiang. 2004. Identification of mine pollution of Dexing Copper Mine with hyperspectral remote sensing. Earth Science-Journal of China University of Geosciences, 29(1).

[30]Qi Xiaoying. \& Yan Mingxing. 2007. Application of multi-temporal remote sensing data in monitoring of mine expansion dynamics. Remote Sensing for Land \& Resources, 73(3). 